P23 (continued)

years;) at two public and two private universities in the New York City area.

Outcome Measures and Analysis: Frequencies, t-tests, and correlational analyses were performed to understand the changes in food consumption and reasons for the change. Multiple regression analysis was performed to predict attitudes toward the U.S. food system.

Results: Students eat more processed foods since coming to the U.S. The longer they stay $(\mathrm{t}=-2.74, \mathrm{p}<.01)$, the more acculturated they become $(\mathrm{r}=.173, \mathrm{p}<.001)$, and the more processed foods they eat. Reasons are convenience, wide availability, and good taste. They eat less whole foods since coming to the U.S. Reasons are time constraints, inconvenience, and expensive price. They make their choices based on nutrition, convenience, and price, but have little concern about ethics and the environment. When attitudes are favorable toward convenience, they choose more processed foods $(\mathrm{F}=3.96, \mathrm{p}<.05)$. When attitudes are favorable toward environmental concerns $(\mathrm{F}=7.03$, $\mathrm{p}<.01)$ and food safety issues $(\mathrm{F}=6.22, \mathrm{p}<.05)$, they choose more whole foods.

Conclusions and Implications: Overall, students choose foods mostly based on nutrition, health, and price and least due to environmental concerns. Therefore, raising students' awareness of food system issues when providing nutrition education is needed.

Funding: None

\section{P24 Knowledge, Perception, and Risk Reducing Behaviors Among Female College Students with Family History of Osteoporosis}

Krishna Patel, MS, RD, kpatel14@kent.edu, Kent State University, 6817 Thornwood Street, Canton, $\mathrm{OH} 44718$; Eun-Jeong $\mathrm{Ha}$, PhD, Kent State University

Objective: Family history of osteoporosis (FHO) has been recognized as one of the most important risk factors for osteoporosis development. The objective of this study was to compare modifiable risk factors, knowledge and perception about osteoporosis and risk reducing behaviors between female college students with FHO and without FHO.

Study Design, Setting, Participants: A convenience sample of 579 female college students attending a Midwestern state University were recruited. Participants completed an online survey with 67 potential questions.

Outcomes Measured and Analysis: Knowledge, perception, risk perception of osteoporosis, and perception in risk reducing behaviors were analyzed through independent t-tests. Chi-square test was performed to analyze differences in modifiable risks of osteoporosis including calcium intake, sun exposure, smoking, physical activity, and beverage consumption in two groups.

Results: Overall, $16.4 \%$ of respondents reported a FHO. Participants with FHO had significantly better knowledge, higher perception and risk perception of osteopo- rosis. They also perceived they were actively practicing osteoporosis risk reducing behaviors $(\mathrm{p} \leq 0.05)$. However, no significant difference was observed in preventative behaviors against osteoporosis including calcium intake, physical activity, caffeinated beverage consumption, sun exposure and smoking between the two groups.

Conclusions and Implications: Students with FHO had more knowledge about osteoporosis, and had higher perception and risk perception of osteoporosis, however were not engaging in adequate preventive behaviors. This study emphasizes the importance of intervention to reduce the disparity between actual and perceived engagement in preventive behaviors against osteoporosis in this population. Electronic reminders such as daily intake/activity trackers can be an effective tool to provide users with a consistent way of monitoring their intake and activities.

Funding: None

\section{P25 A Mixed-Methods Approach to Evaluate the Effectiveness of Rural Community Health Coalitions: Comparing Coalition Capacity and Social Network Analysis}

Jennifer Mansfield, BS, jmansfi@purdue.edu,

Purdue University, 700 West State Street,

West Lafayette, IN 47907; Lindley McDavid, PhD, Purdue

University; Donna Vandergraff, MS, RD; Krystal Lynch, PhD;

Dennis Savaiano, $P h D$

Objective: To determine how multiple indicators of coalition capacity (robustness, strength, sustainability, and growth) may predict effectiveness of community health coalitions in rural settings.

Study Design, Setting, Participants, Intervention: Community coalitions in two rural Midwestern counties focusing on building capacity to reduce obesity participated in this study. Local community facilitators were hired to assist coalitions in forming partnerships, creating mission statements and goals, and identifying/ addressing community needs. We are taking a novel mixed-methods approach to assess associations across indicators of coalition capacity within and between counties to determine which variables are most salient to coalition effectiveness. Participants are completing interviews and surveys over a two-year period that measure the study variables.

Outcome Measures and Analysis: Indicators of coalition capacity are being assessed using social network analysis and factor analysis to determine how assessment approaches converge. We are comparing network robustness, density, and centrality to coalition growth, health, and sustainability.

Results: We expect to demonstrate the unique and shared power of coalition capacity indicators and determine which indicators may serve as instrumental predictors of coalition effectiveness. 
P25 (continued)

Conclusions and Implications: Comparing quantitative network parameters (i.e. density and centrality) with other capacity building assessments will allow us to determine how specific modifications to the network (e.g. increasing density and/or centrality or restructuring the network geometry) may predict and improve coalition effectiveness over time. This novel methodology can be used for long-term evaluation and planning for local, state, and regional coalitions.

Funding: Center for Disease Control and Prevention

\section{P26 "Village Lunch Table" for the Korean Elderly in Rural Areas - A Pilot Program}

Yourim Choi, BA, RD, Myongji University;

Youngmi Lee, PhD; Youngsuk Lim, PhD, RD;

Kyungeun Lee, PhD, RD, Seoul Women's University; Changhee You,PhD, RD; Haeryun Park,PhD, RD, hrpark@ mju.ac.kr, Myongji University, 116 Myongji-ro, Cheoin$\mathrm{gu}$, Yongin city, Gyeonggi-do, Republic of Korea 17058

Objective: To develop and deliver a comprehensive nutrition program to improve the dietary quality and health of the elderly living in rural areas in South Korea.

Theory, Prior Research, Rationale: Compared to the elderly in urban areas, the elderly in rural areas are more vulnerable to inferior diets and loneliness, affected by the lack of public infrastructure, budget constraints, tendency to live alone, lack of health literacy, and geographical distances, etc.

Description: Forty different menus and nutrition education materials were developed and implemented, providing two free meals weekly for 10 weeks at 50 villages (delivered to about 1650 elderly). The cooking facilities of the community centers of each village were used. Helpers preparing meals and the elderly people were preeducated with nutrition materials on the five basic food groups, food hygiene, and dietary treatment for diabetes and hypertension.

Evaluation: The evaluation tools were surveys on recipient satisfaction, and diet quality comparison of meals provided versus the previous day's meals measured by the number of food (DVS) and food groups (GMVFD) consumed.

Conclusion and Implications: The satisfaction score was very high and the diet proved to be enhanced. This program of integrated meal service and nutrition education should be expanded to prevent the rural elderly from under nutrition and loneliness and to improve their health and quality of life.

Funding: Korean Ministry of Agriculture, Food, and Rural Affairs in 2016

\section{P27 WITHDRAWN}

\section{P28 Assessing Implementation Fidelity of Go for Green ${ }^{\circledR}$, a Military Performance Nutrition Initiative}

Alyssa Coleman, MPH, BS, CPH, alyssa.m.coleman2.ctr@ mail.mil, US Army Public Health Center, 5158 Blackhawk Road, APG-Edgewood, MD 21010; Chizoba Chukwura, MPH, BS, CPH, US Army Public Health Center; Karen Langlois, RDN, LD; Alyssa Dimatulac, BS; Bethany Belanger, PhD, RD, MAJ, LD, CSCS, Madigan Army Medical Center

Objective: To assess implementation of evidence-based nudging strategies in military dining facilities (DFACs), obtain feedback to refine future program implementation and examine changes to DFAC environments.

Target Audience: Since 2011, DFACs participated in the original version of Go for Green ${ }^{\circledR}(G 4 G)$, a military performance-nutrition program, utilizing standard food labels and print materials. In 2015, G4G was revamped with added nudging strategies and piloted over six months at seven DFACs within five continental U.S. Army installations.

Theory, Prior Research, Rationale: Food consumer research shows nudging empowers consumers to make healthier selections. Traffic light labeling is shown to effectively improve consumers' identification and selection of healthier items. Further studies show interventions using labeling in combination with choice architecture resulted in greater impact.

Description: A pre and post (photographs of DFAC serving areas) and post only (staff surveys, management interview) assessment of G4G implementation fidelity, barriers, facilitators, and environmental changes in DFACs.

Evaluation: After six months, results showed a 7\% increase in the proportion of food items labeled with G4G cards and a $4 \%$ increase in the level of incorporation of choice architecture indicators over baseline. Evaluation participants noted operational (e.g., staff attitudes and beliefs) and strategic (e.g., accountability, regulatory, and budget constraints) barriers to G4G implementation. Positive perceptions of G4G, program knowledge, and buy-in to partnering initiatives enhanced implementation success.

Conclusions and Implications: $G 4 G$ leadership is currently improving implementation guidance and introducing metrics to monitor program fidelity based on evaluation findings. Evaluation results may inform successful and sustainable nudging strategy implementation in military and comparable civilian food service environments.

Funding: None 